\title{
Transient Functional Suppression and Facilitation of Japanese Ideogram Writing Induced by Repetitive Transcranial Magnetic Stimulation of Posterior Inferior Temporal Cortex
}

\author{
Yoshino Ueki, ${ }^{1}$ Tatsuya Mima, ${ }^{1}$ Kimihiro Nakamura, ${ }^{2}$ Tatsuhide Oga, ${ }^{1}$ Hiroshi Shibasaki, ${ }^{1,3}$ Takashi Nagamine, ${ }^{1}$ and \\ Hidenao Fukuyama ${ }^{1}$ \\ ${ }^{1}$ Human Brain Research Center, Kyoto University Graduate School of Medicine, Sakyo-ku, Kyoto 606-8507, Japan, ${ }^{2}$ Section of Speech Physiology, \\ Department of Speech and Cognitive Neuroscience, University of Tokyo, Bunkyo-ku, Tokyo 153-8914, Japan, and ${ }^{3}$ Department of Neurology, Takeda \\ General Hospital, Fushimi-ku, Kyoto 601-1495, Japan
}

\begin{abstract}
The Japanese writing system is unique in that it is composed of two different orthographies: kanji (morphograms) and kana (syllabograms). The retrieval of the visual orthographic representations of Japanese kanji is crucial to the process of writing in Japanese. We used low-frequency repetitive transcranial magnetic stimulation (rTMS) to clarify the functional relevance of the left and right posterior inferior temporal cortex (PITC) to this process in native Japanese speakers. The experimental paradigms included the mental recall of kanji, kana-to-kanji transcription, semantic judgment, oral reading, and copying of kana and kanji. The first two tasks require the visualization of the kanji image of the word. We applied $0.9 \mathrm{~Hz}$ rTMS (600 total pulses) over individually determined left or right PITC to suppress cortical activity and measured subsequent task performance. In the mental recall of kanji and kana-to-kanji transcription, rTMS over the left PITC prolonged reaction times (RTs), whereas rTMS over the right PITC reduced RTs. In the other tasks, which do not involve the mental visualization of kanji, rTMS over the left or right PITC had no effect on performance. These results suggest that the left PITC is crucial for the retrieval of the visual graphic representation of kanji. Furthermore, the right PITC may work to suppress the dominant left PITC in the neural network for kanji writing, which involves visual word recognition.
\end{abstract}

Key words: Japanese ideogram writing; kanji; visual word recognition; posterior inferior temporal cortex; PITC; repetitive transcranial magnetic stimulation; rTMS; interhemispheric neural networks

\section{Introduction}

In the West, many patient studies, beginning with that of Déjerine, have established that lesions of the left angular gyrus produce writing disturbances associated with reading impairments (alexia with agraphia) (Dejerine, 1891; Geschwind, 1965). Later, another type of agraphia was described: damage to the junction of the left angular gyrus and the parieto-occipital lobule produces lexical agraphia. This damage is attributable to a disruption in the whole-word retrieval system necessary to read orthographically irregular words (Roeltgen et al., 1983; Roeltgen and Heilman, 1984; Roeltgen, 1993). The study of functional differences in left

\footnotetext{
Received Feb. 24, 2006; revised June 27, 2006; accepted June 29, 2006.

This study was partly supported by Ministry of Education, Culture, Sports, Science, and Technology of Japan Grants-in-Aid for Scientific Research on Priority Areas 18019020 (Integrative Brain Research; to T.M.) and 18020014 (System Study on Higher-Order Brain Functions; to H.F.), and a research grant (2004) from the Suzuken Memorial Foundation (T.M.). We thank Dr. Yoshihiro Takayama and Dr. Mark Hallett for useful discussions.

Correspondence should be addressed to Dr. Tatsuya Mima, Human Brain Research Center, Kyoto University Graduate School of Medicine, 54 Shogoin Kawahara-cho, Sakyo-ku, Kyoto 606-8507, Japan. E-mail: mima@kuhp.kyoto-u.ac.jp.

D0I:10.1523/JNEUROSCI.0846-06.2006

Copyright $\odot 2006$ Society for Neuroscience $\quad 0270-6474 / 06 / 268523-08 \$ 15.00 / 0$
}

hemisphere writing systems has thus, as yet, been based solely on studies of Western languages.

The Japanese writing system is unique in that it is composed of two different orthographies: kanji (morphograms) and kana (syllabograms). Because kanji characters are complex graphic forms that are associated with semantics as well as multiple phonetic representations, kanji writing requires the selection of a contextually appropriate lexical item from among several homophone candidates stored in orthographic memory. In contrast, kana characters have only phonological meanings, comparable with the Western alphabet.

Japanese patients with lesions in the left angular gyrus show alexia of kana with agraphia of both kana and kanji, whereas lesions in the left posterior inferior temporal cortex (PITC) result in alexia with agraphia for kanji only (Yamadori, 1975, 2000; Kawamura et al., 1987; Kawahata et al., 1988; Soma et al., 1989; Sakurai et al., 1994). Recent functional neuroimaging studies have shown that language tasks involving kanji, especially kanji writing and the mental retrieval of images, activate the left PITC (Tokunaga et al., 1999; Nakamura et al., 2000; Sakurai et al., 2000). Thus, kanji and kana writing systems may involve different subprocesses in the left hemisphere. The left PITC may be 
involved in kanji writing processing, particularly the subprocess of accessing the visual graphic kanji images stored in long-term memory (Iwata, 1984).

Low-frequency repetitive transcranial magnetic stimulation (rTMS) at $\sim 1 \mathrm{~Hz}$ can produce focal and transient disruption of cortical function that lasts beyond the duration of the stimulus train (Chen et al., 1997; Walsh and Cowey, 1998; Pascual-Leone et al., 1999). This inhibitory effect of rTMS is caused by the transient suppression of cortical excitability in the focal region in which rTMS was delivered (Chen et al., 1997; Kosslyn et al., 1999; Satow et al., 2003). This technique has been used to show whether a given brain region is essential for a particular cognitive process (Walsh and Rushworth, 1999). Thus, based on neuroimaging studies, we can evaluate the relationship between the targeted cortical region and a particular type of brain processing.

By using low-frequency rTMS in normal volunteers, we examined whether the left PITC is crucial to the retrieval of visual orthographic kanji representations, which is a process necessary during writing. Second, we investigated the potential role of the right PITC in writing kanji. This latter issue has rarely been addressed, and its elucidation could contribute to the development of new therapies for aphasia (Martin et al., 2004).

\section{Materials and Methods \\ Subjects}

Seventeen healthy volunteers (13 males and 4 females, aged $27.5 \pm 5.0$ years, mean $\pm \mathrm{SD}$ ) were involved in the study. All were native Japanese speakers and right-handed, as confirmed by the Edinburgh inventory. None had a history of psychiatric or neurological disease. Subjects were fully instructed regarding the details of the study and gave written informed consent before the experiment. The protocol was in accordance with the guidelines determined by the Ethics Committee of Kyoto University Graduate School and Faculty of Medicine (protocol number 267).

\section{Word stimuli}

One hundred twenty-eight two-character compound kanji words were selected from the fundamental vocabulary for the teaching of the Japanese language. When spelled in kana, the words consisted of two to six kana characters. Kana scripts corresponding to the selected kanji words were also used as stimuli. Half of the 128 words represented concrete nouns and the other half abstract nouns. There were no homophones. The level of concreteness and imagery of most words exceeded level 6 based on the 7-level scales of concreteness and imagery (Ogawa and Inamura, 1974). In most cases, the lexical frequency of the words exceeded 500 per 1,000,000. All of the words used in this study are conventionally spelled in kanji in daily life. A set of 32 words in kanji and 32 words in kana were randomly selected from the 128 words for each session. Each word was presented in white, horizontally in the center of a black screen located $100 \mathrm{~cm}$ in front of subjects (visual angle of $0.9-2.6^{\circ}$ ). Each kanji or kana word was presented only once during a session. Each stimulus was presented for $400 \mathrm{~ms}$, and the interstimulus interval (onset to onset) was $2500 \mathrm{~ms}$. In each session, subjects had to perform one of the six predetermined tasks described below. They were instructed to perform the tasks as quickly and accurately as possible, immediately after each stimulus appeared.

\section{Behavioral tasks}

Each subject completed six behavioral tasks, including the mental recall of kanji orthography, kana-to-kanji transcription, semantic judgment, oral reading, and copying kana and kanji (Fig. 1). These tasks are similar to those used in a previous study (Nakamura et al., 2000).

a: Mental recall of kanji. Some kanji characters are composed of two radicals that are juxtaposed horizontally (L-R type), whereas others are not arranged according to this rule (non-L-R type). In this task, subjects responded by pressing one of two buttons: button 1 or 2 , with the index or middle finger, respectively, of the right-hand. We instructed subjects to mentally transcribe each kana word into kanji script and press button

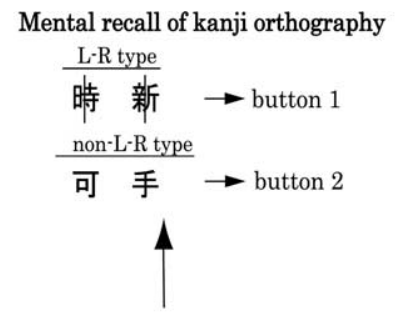

Kana-to kanji transcription
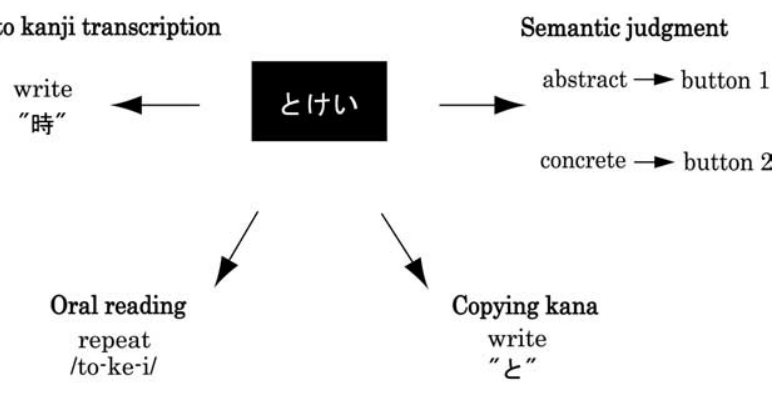

\author{
.
}




\section{Left PITC}
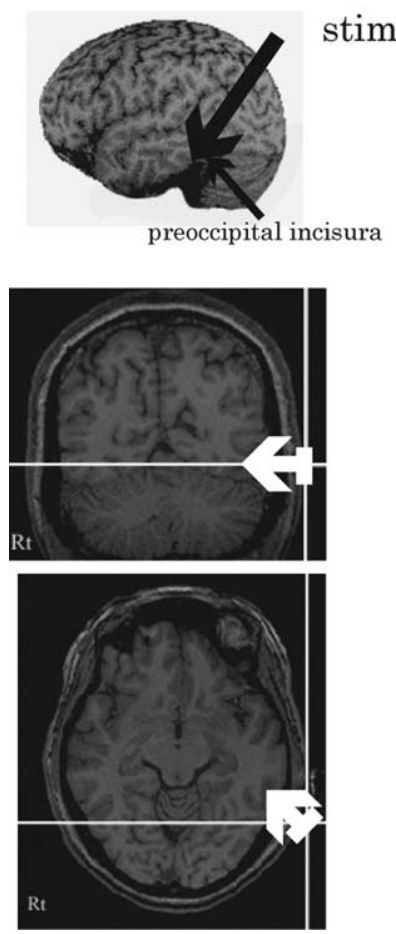

Figure 2. TMS stimulation sites for the left PITC and right PITC. The top, middle, and bottom panels show the three-dimensional surface brain reconstructions, and coronal and axial slices, respectively, of the MRI scans from one subject. In the top panels, the black thick arrows represent the coil stimulation site, and the direction of the arrows indicates the current flow induced by TMS in the brain on the three-dimensional MRI. The thin black arrows show the preoccipital incisura. In each of the middle and bottom panels, two perpendicular white lines are drawn, the intersection of which indicates the coil contact at which the induced electric field is at its maximum. The white thick lines indicate the orientation of the stimulation coil, and the white arrows represent the magnetic stimulation sites. Lt, Left; Rt, right.

ments 1-3 were $2000 \mathrm{~Hz}$. TMS was performed with a Magstim Super Rapid Magnetic Stimulator (biphasic, pulse width of $250 \mu \mathrm{s}$ ) (Magstim, Whitland, Dyfed, UK) using an air-cooled figure-eight coil composed of two loops with an external diameter of $70 \mathrm{~mm}$ (maximum magnetic field strength, 0.93 tesla). The coil was placed over the optimal position in the left primary motor cortex to elicit the best motor response in the right FDI muscle. The resting motor threshold was defined as the lowest stimulus intensity required to elicit motor-evoked potentials with a peak-topeak amplitude of $>50 \mu \mathrm{V}$ in the right FDI muscle in at least 5 of 10 trials (Rossini et al., 1994). In the present study, the rTMS stimulus intensity over the target area was fixed at $90 \%$ of the resting motor threshold for each individual subject and kept constant throughout the intervention. This TMS intensity was used to maximize the chance of local disruption of neural function without producing excessive discomfort (Mottaghy et al., 2002).

\section{TMS stimulation site}

TMS was applied to a region corresponding to the PITC of the left or right hemisphere on the basis of an anatomical magnetic resonance image of each subject. The magnetic resonance imaging (MRI) was conducted on a 3 or 0.2 tesla whole-body scanner equipped with a volume head coil [ 3 tesla (Trio; Siemens, Erlangen, Germany) or 0.2 tesla (Signa unit; General Electric, Milwaukee, WI)], and T1-weighted axial images were obtained. The TMS stimulation site was identified with a frameless stereotaxic system Brainsight (Rogue Research, Montreal, Quebec, Canada), which facilitates the positioning of the coil and allows the simultaneous visualization of the coil on a magnetic resonance image (Fig. 2). In the three-dimensional magnetic resonance image, to target the left PITC, we located the area $1 \mathrm{~cm}$ rostral to the left preoccipital incisura; this is where peak activation was identified in our previous language study with functional MRI (fMRI) (Nakamura et al., 2000). With regard to the right PITC, we targeted the area $1 \mathrm{~cm}$ rostral to the right preoccipital incisura, because right PITC activation has been identified previously in the symmetrical right-hemisphere homolog of the left PITC (Cohen et al., 2002). On the scalp, the center of the coil position for PITC stimulation was located $2.5-3.0 \mathrm{~cm}$ dorsal and $9.0-12.0 \mathrm{~cm}$ lateral to the inion in the left hemisphere and $2.0-3.0 \mathrm{~cm}$ dorsal and $8.0-11.0 \mathrm{~cm}$ lateral to the right hemisphere inion.

\section{Experimental procedures}

The subjects were seated in an armchair with their arms placed on the armrests. In an experiment taking place during one day, rTMS was given at one target site and subjects performed two different tasks of all six tasks (tasks X and XX) before (pre-) and just after (post-) rTMS (total of four sessions) (Fig. 3). The behavioral parameters of the two different prerTMS tasks were used to measure the baseline performance of each task. The order of the two tasks in pre-rTMS and post-rTMS sessions was randomized across subjects to cancel the order effect. Because one session ( 32 trials) lasted $<2 \mathrm{~min}$ and there was no interval between sessions 3 and 4 in post-rTMS, two sessions in post-rTMS (sessions 3 and 4 ) were completed within $5 \mathrm{~min}$ after rTMS had finished. This is important because the carryover effects of rTMS last half as long as the stimulation period (Chen et al., 1997; Muellbacher et al., 2000). The order that the two different tasks were given in pre-rTMS and post-rTMS was counterbalanced across subjects.

\section{Experiment 1: stimulation of the PITC}

To evaluate the functional relevance of the left and right PITC to the retrieval of the visual orthographic representation of kanji, rTMS was applied to the left or right PITC at a frequency of $0.9 \mathrm{~Hz}$ for $11 \mathrm{~min}$ (total of 600 pulses). The six tasks were divided into three sets of two tasks each: set 1 consisted of task a (mental recall of kanji) and task c (semantic judgment); set 2 was task b (kana-to-kanji transcription) and task d (oral reading); and set 3 was tasks e and $\mathrm{f}$ (copying kana and kanji, respectively). As described above, rTMS was given at either the left or right PITC, and one of the three sets of tasks was assigned to each subject. Therefore, the subjects performed two different tasks (tasks X and XX), each of which had pre-rTMS and post-rTMS sessions; this resulted in four sessions in total in one day (Fig. 3). In the experiment on the following day, rTMS was given to the opposite PITC while subjects performed the same task set. Ten subjects were involved in only one of each task set over $2 \mathrm{~d}$ : set 1 consisted of nine males and one female, aged $28.1 \pm 6.2$ years; set 2 was seven males and three females, aged $28.1 \pm 5.5$ years; and set 3 was seven males and two females, aged $26.1 \pm 4.9$ years. Some subjects repeated several sets of two tasks each in a different day each, i.e., seven subjects participated in two sets (four tasks in total), and four subjects completed three sets of tasks (six tasks total). To exclude the possible carryover effect, we performed the rTMS only once a day. Any two successive experiments were performed at least $3 \mathrm{~d}$ apart. The order of the participation in each of the three sets and the target sites for rTMS were randomized across subjects.

\section{Experiment 2: stimulation of a control site in the left hemisphere} To investigate the regional specificity of the effect of rTMS over the left PITC, we applied rTMS over another location in the left hemisphere. We randomly selected six subjects from among the 10 subjects recruited for experiment 1 (five males and one female, aged $29.2 \pm 6.2$ years). All six or four subjects performed the same tasks, a and $b$ (mental recall of kanji or kana-to-kanji transcription, respectively), as they had done in experiment 1 . They participated in experiment 2 at least 1 month apart from experiment 1 . We targeted a region $2 \mathrm{~cm}$ dorsal from the point of PITC stimulation on the scalp, whose coil position was determined using Brainsight. Because the responses in the mental recall of kanji and kanato-kanji transcription tasks were significantly affected by rTMS over the left PITC in experiment 1 , we chose only these tasks for experiment 2. The task order (two tasks) was randomized across subjects.

\section{Experiment 3: sham stimulation over the right PITC}

To confirm the possible contribution of learning to the facilitatory effect of rTMS, we performed control sham stimulation over the right PITC in 
seven subjects, five of whom were randomly selected from among the subjects recruited for experiment 1 (five males and two females, aged $30.1 \pm 3.4$ years). Five or three subjects performed the same tasks, a or b, as the one in experiment 1 . They participated in experiment 3 at least 1 month apart from experiment 1. Under the sham stimulation, a sham coil was positioned over the right PITC, and it produced a discharge click similar to that produced by the real rTMS, without producing a magnetic field. Subjects performed the mental recall of kanji and the kana-to-kanji transcription tasks in a single day. The order of the two tasks was randomized across subjects.

\section{Analysis}

For each trial, both response accuracy and behavioral performance were assessed. In the mental recall of kanji and the semantic judgment tasks, RT was measured by the timing of the button press after the stimulus onset. During oral reading, the subject's voice was recorded via a microphone, and the onset time of vocalization was used as the RT. In kana-to-kanji transcription and when copying kana and kanji script, the writing was recorded using a force transducer. The onset time of writing pressure was used as the RT. The responses of each subject were monitored with a video camera and scored both on-line and off-line.

For each session, the mean RT was calculated, and response accuracy was expressed as the percentage of correct answers (RA\%). Trials in which there was no response for $>2 \mathrm{~s}$ were marked as errors and excluded from the mean RT analysis.

To evaluate the effect of rTMS over the left and right PITC in experiment 1 , the changes in mean RT and RA\% were evaluated using a threefactor mixed-model ANOVA, task (tasks a-f), time (pre-rTMS or postrTMS), and hemisphere (left or right PITC) as the main factors. The variance of the subjects was treated as a random effect. If the effect was significant, a post hoc test was performed to explore the strength of the main effects and patterns of interactions between factors. In experiments 2 and 3 , to evaluate the different effects of rTMS over the site more dorsal to the left PITC and sham stimulation over the right PITC, changes in mean RT and RA\% were examined for each session using paired $t$ tests. The threshold of significance was set at $p<0.05$.

\section{Results}

The resting motor threshold for the right FDI was $75.1 \pm 8.1 \%$ of the maximal stimulator output. The actual stimulus intensity of rTMS was $67.8 \pm 5.9 \%$ (mean $\pm \mathrm{SD})$.

Experiment 1: the effect of rTMS over the left or right PITC in all six tasks (Table 1)

Reaction times

Looking at the between-subjects effects, the three-factor ANOVA showed a significant main effect of task $\left({ }^{*} p<0.001\right)$ but no significant main effect of side $(p=0.89)$ and time $(p=0.25)$. There was significant task by side interaction $\left({ }^{*} p=0.003\right)$, side by time interaction $\left({ }^{*} p=0.001\right)$, task by time interaction $\left({ }^{*} p=\right.$ $0.006)$, and task by side by time interaction $\left({ }^{*} p<0.001\right)$. Although there was no significant effect of the time contrast, the time factor showed interactions with task by side. Thus, we examined separate two-factor ANOVAs for each task independently, with side (left or right) and time (pre-rTMS or postrTMS) as the between-subjects factor. In task a, mental recall of kanji, the ANOVA revealed a significant contrast for a time by side interaction $\left({ }^{*} p=0.001\right)$ but no significant main effect of time $(p=0.51)$ and side $(p=0.08)$. The post hoc analysis comparing the effect of time revealed significantly prolonged RTs after rTMS in the left PITC $\left({ }^{*} p=0.01\right)$ but significantly short-
Table 1. Mean RTs and RA\% in each of the six tasks before and after TMS over the left and right PITC

\begin{tabular}{llllll}
\hline & \multicolumn{2}{l}{ Left PITC } & & \multicolumn{1}{l}{ Right PITC } \\
\cline { 2 - 3 } \cline { 5 - 6 } & Pre-TMS & Post-TMS & & Pre-TMS & Post-TMS \\
\hline RTs (ms) & & & & \\
a & $980 \pm 180$ & $1080 \pm 170^{*}$ & & $980 \pm 140$ & $910 \pm 120^{*}$ \\
b & $700 \pm 110$ & $870 \pm 120^{*}$ & & $750 \pm 160$ & $660 \pm 110^{*}$ \\
c & $950 \pm 140$ & $910 \pm 130$ & & $960 \pm 170$ & $900 \pm 170$ \\
d & $490 \pm 70$ & $490 \pm 60$ & & $560 \pm 110$ & $500 \pm 60$ \\
e & $390 \pm 50$ & $370 \pm 50$ & & $380 \pm 50$ & $360 \pm 60$ \\
f & $430 \pm 70$ & $420 \pm 40$ & & $440 \pm 60$ & $410 \pm 60$ \\
RA\% & & & & \\
a & $94.6 \pm 6.3$ & $89.0 \pm 5.1^{*}$ & & $92.4 \pm 5.7$ & $94.1 \pm 4.0$ \\
b & $97.2 \pm 3.1$ & $97.8 \pm 2.6$ & & $98.1 \pm 2.2$ & $98.8 \pm 2.2$ \\
c & $90.0 \pm 3.8$ & $91.3 \pm 4.1$ & & $90.3 \pm 4.5$ & $91.8 \pm 5.0$ \\
d & 100 & 100 & & 100 & 100 \\
e & 100 & 100 & & 100 & 100 \\
f & 100 & 100 & & 100 & 100
\end{tabular}

${ }^{*} p$ values were produced using post hoc analyses comparing the mean level of RTs and RA\% in each task between pre-rTMS and post-rTMS. a, Mental recall of kanji; b, kana-to-kanji transcription; $c$, semantic judgment; $d$, oral reading; e, copying kana; $f$, copying kanji.

ened RTs after rTMS in the right PITC $\left({ }^{*} p=0.04\right)$. In task $b$, kana-to-kanji transcription, the ANOVA demonstrated a significant contrast for side $\left({ }^{\star} p=0.02\right)$, significant time by side interaction $\left({ }^{\star} p=0.001\right)$, but no significant effect of time $(p=0.11)$. The post hoc analysis comparing the effect of time demonstrated significantly prolonged RTs after rTMS in the left PITC $\left({ }^{*} p=\right.$ $0.003)$ but significantly shortened RTs after rTMS in the right PITC $\left({ }^{*} p=0.02\right)$. In task $c$ (semantic judgment), task d (oral reading), task e (copying kana), and task $\mathrm{f}$ (copying kanji), ANOVAs revealed no significant contrast for time by side interaction (c, semantic judgment, $p=0.65$; d, oral reading, $p=0.22$; e, copying kana, $p=0.47$; and $\mathrm{f}$, copying kanji, $p=0.74$ ).

\section{Response accuracy}

Several subjects claimed that they momentarily "forgot" letters during task a (mental recall of kanji) and task b (kana-to-kanji transcription) after rTMS.

Looking at the between-subjects effects, the three-factor ANOVA showed a significant main effect of task $\left({ }^{*} p<0.001\right)$ but no significant main effect of side $(p=0.10)$ and time $(p=0.94)$. There was significant side by time interaction $\left({ }^{*} p=0.03\right)$ and task by side by time interaction $\left({ }^{*} p=0.03\right)$ but no significant task by time $(p=0.10)$ and task by side $(p=0.80)$ interaction. Although there was no significant effect of the time contrast, the time factor showed interactions with task by side. Thus, we examined separate two-factor ANOVAs for each task indepen- 


\section{(A) Control site (B) Sham stimulation}

(a) Mental recall
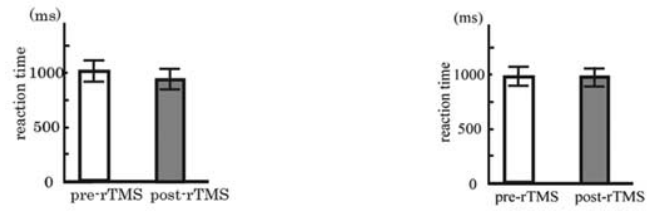

(b) Kana to kanji transcription
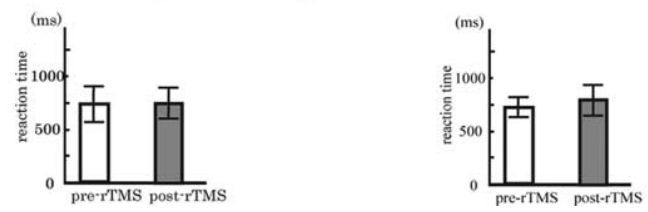

Figure 4. A, Effect of rTMS over the control site (2 cm dorsal to the left PITC) on RTs in the mental recall of kanji and kana-to-kanji transcription tasks. In task a (mental recall of kanji) and task b (kana-to-kanji transcription), rTMS over the control site had no effect on RTs. B, Effect of sham stimulation over the right PITC on RTs in the mental recall of kanji and kana-to-kanji transcription tasks. In both tasks $a$ and b, there were no effects on RTs after sham stimulation.

dently, with side (left or right) and time (pre-rTMS or postrTMS) as the between-subjects factor. In task a, mental recall of kanji, the ANOVA revealed a significant contrast for a time by side interaction $\left({ }^{*} p=0.02\right)$ but no significant main effect of time ( $p=0.27$ ) and side $(p=0.22)$. The post hoc analysis comparing the effect of time revealed significantly decreased RA\% after rTMS in the left PITC $\left({ }^{*} p=0.03\right)$ but no effect in the right PITC ( $p=0.47$ ). In the task b (kana-to-kanji transcription) and task $\mathrm{c}$ (semantic judgment), the ANOVA revealed no significant contrast for time by side interaction (b, kana-to-kanji transcription, $p=1.00 ; c$, semantic judgment, $p=1.00)$.

\section{Experiment 2: effect of rTMS over the control site in the left hemisphere}

When rTMS was delivered to the site $2 \mathrm{~cm}$ dorsal to the left PITC, the RTs after rTMS were not significantly different from those measured pre-rTMS for task a (mental recall of kanji, $1050 \pm 140$ ms pre-rTMS; $950 \pm 110$ ms post-rTMS; $p=0.12$ ) or task $b$ (kana-to-kanji transcription, $750 \pm 160 \mathrm{~ms}$ pre-rTMS; $750 \pm 130$ ms post-rTMS; $p=0.92$ ) (Fig. $4 A$ ). There were no effects on the RA\% of rTMS of the control site for either task a $(96.9 \pm 5.1 \%$ pre-rTMS; $95.6 \pm 5.1 \%$ post-rTMS; $p=0.84)$ or task $\mathrm{b}(99.5 \pm$ $1.3 \%$ pre-rTMS; $99.0 \pm 2.6 \%$ post-rTMS; $p=0.36$ ).

Experiment 3: effect of sham stimulation over the right PITC The RTs were not changed by sham stimulation for either task a (mental recall of kanji orthography, $990 \pm 100 \mathrm{~ms}$ pre-rTMS; $980 \pm 100$ ms post-rTMS; $p=0.90$ ) or task b (kana-to-kanji transcription, $740 \pm 80 \mathrm{~ms}$ pre-rTMS; $790 \pm 120 \mathrm{~ms}$ post-rTMS; $p=0.62$ ) (Fig. $4 B$ ). The RA\% scores were also unaffected by sham stimulation (task a, $93.7 \pm 4.4 \%$ pre-rTMS, $94.7 \pm 3.9 \%$ post-rTMS, $p=0.62$; task b, $98.7 \pm 2.4 \%$ pre-rTMS, $99.1 \pm 2.3 \%$ post-rTMS, $p=0.36$.

Regarding the variability of the baseline performance of tasks $\mathrm{a}$ and $\mathrm{b}$ across subject groups, there were no significant differences between experiments 1 and 2 or between experiments 1 and 3 for both RTs and RA\%: for experiment 1 versus $2, p=0.45$ for RTs and $p=0.82$ for RA $\%$; for experiment 1 versus $3, p=0.89$ for RTs and $p=0.76$ for RA\% in task a; for experiment 1 versus 2 , $p=0.41$ for RTs and $p=0.11$ for RA $\%$; for experiment 1 versus $3, p=0.41$ for RTs and $p=0.31$ for RA\% in task b (examined by Student's $t$ test).

\section{Discussion}

The present study showed transient functional suppression and facilitation effects on Japanese kanji writing induced by lowfrequency rTMS over the left and right PITC, respectively. The rTMS over the left PITC induced more errors and longer RTs in the tasks that required the visualization of kanji (the mental recall of kanji and kana-to-kanji transcription), suggesting that the left PITC is likely to be crucial for the mental retrieval of the graphic representation of kanji. In contrast, rTMS over the right PITC reduced RTs in the mental recall of kanji and kana-to-kanji transcription tasks, indicating that the nondominant PITC might work as a suppressor in the neural network required for writing kanji.

\section{Functional role of the left PITC in kanji writing}

In the mental recall of kanji task, we found a significantly lower RA\% after rTMS over the left PITC. Additionally, rTMS over the left PITC resulted in longer RTs during the mental recall of kanji and kana-to-kanji transcription, which reflect the surplus time necessary to process these tasks as a result of the transient functional disruption of the left PITC. Because there is a trade-off between speed and accuracy in reaction time tasks, the longer RTs in these tasks after rTMS might have the same functional meaning as increased error rates. Because kana-to-kanji transcription is a highly skilled but almost automated procedure for normal Japanese adults, the transient functional depression of PITC could be easily compensated for by a small increase in RT, without leading to an increase in errors. Detailed psycholinguistic analysis based on the symptoms and lesion sites suggest that kanji-selective agraphia arises from an impaired access to visual graphic kanji images stored in long-term memory. This writing disorder is thought to be analogous to the lexical agraphia seen in Western languages (Roeltgen et al., 1983; Roeltgen and Heilman, 1984). Our findings complement the conventional view from lesion and fMRI studies that this area participates in the processing of kanji writing through orthographic retrieval, even with no accompanying overt motor execution (Soma et al., 1989; Nakamura et al., 2000).

In contrast, the RTs and RA\% scores for the oral reading and semantic judgment tasks were not affected by rTMS, suggesting that the left PITC is not involved in the phonological or semantic aspects of language processing. This finding is consistent with a previous fMRI study showing that the left PITC is not activated during these two tasks (Nakamura et al., 2000).

When copying kana and kanji, the RTs and RA\% scores were unaffected by rTMS over the left PITC. This finding is in good agreement with those of the lesion and cortical electrical stimulation studies of this region, which report that copying kana and kanji is not impaired (Soma et al., 1989; Usui et al., 2003). Because Japanese subjects can write kanji characters quite automatically through repeated practice, such visual kanji images may be important for activating the stored graphic motor patterns required for writing. Thus, the orthographic representation of kanji includes the graphomotor representation as well as the visual representation. It is possible that the grapheomotor representations for writing kanji and kana are stored in the frontoparietal network, in which lesions most commonly affect the writing of both forms of script (Iwata, 1984; Nakamura and Kouider, 2003). 
Similarly, our findings showed that the left PITC is not associated with the grapheomotor aspects of writing.

In the mental recall of kanji and the kana-to-kanji transcription tasks, rTMS over a site $2 \mathrm{~cm}$ away (dorsal) from the left PITC had no effects on the RTs or RA\% scores. Thus, the retrieval of the visual graphic form when writing kanji seems to be a function specifically confined to the PITC.

Because effects of rTMS spread trans-synaptically distant regions, spatial resolution becomes lower than the maximum resolution of single-pulse or paired-pulse TMS. Mapping studies within the motor or visual cortex using single-pulse TMS have reported a spatial resolution of $0.5-1 \mathrm{~cm}$ at the scalp surface, but, if using a prolonged train of rTMS, the spread of the effects along both corticocortical and cortico-subcortical connections may well lead to less focal effects (Pascual-Leone et al., 1994; Jahanshahi and Rothwell, 2000; Walsh and Cowey, 2000). Furthermore, several recent studies combining TMS with neuroimaging methods have demonstrated that TMS can produce, in addition to local effects on the directly targeted cortex, transsynaptic effects on distant cortical regions (Lee et al., 2003; Rounis et al., 2005; Valero-Cabre et al., 2005). In the present study, rTMS over a site $2 \mathrm{~cm}$ away from the left PITC and sham stimulation had no effects on the task performance. However, it remains possible that not only the PITC but also through PITCspecific connected brain regions participate in the task related to the retrieval of the visual graphic representation of kanji because of the methodological limitation of rTMS.

For experiments 2 and 3, some subjects performed the same task as the one they had done in experiment 1 . However, because they participated in experiments 2 and 3 at least 1 month apart from experiment 1 , we do not think that the learning effect might make a difference in task performance. Furthermore, we evaluated the change in task performance during baseline conditions (pre-rTMS) between experiments 1 and 2 or between experiments 1 and 3 for both RTs and RA\% and found no significant difference. Thus, the variability of the task performance across subject groups was likely to be negligible in this study.

\section{Functional role of the left PITC in tasks other than kanji writing}

The left PITC, mainly corresponding to Brodmann area 37, constitutes part of the ventral visual pathway involved in the analysis of complex figures and object recognition (Sergent et al., 1992; Moscovitch et al., 1995; Clark et al., 1996; Price et al., 1996; Rosier et al., 1997; Ishai et al., 1999; Stewart et al., 2001; Rossion et al., 2003). In fact, patients suffering from alexia with agraphia of kanji caused by this lesion show only mild naming difficulties (Sakurai et al., 1994). However, our results do not imply that kanji are just complex figures and that Japanese people write kanji as they would draw objects, because kanji characters are strongly associated with semantics and phonology, which are implicitly activated on visual presentation of kanji, as well as visual graphic forms (Thuy et al., 2004). Therefore, the visual recognition of kanji is likely to use a different processing system from that of object recognition, although a similar brain region might be involved in both forms of processing.

Recently, some authors have referred to the left PITC as the "visual word form area" of the brain, although it is still controversial as to whether or not there is a confined area dedicated to word recognition (Cohen et al., 2002). This area is activated whenever subjects read or write printed words, and its function may be to encode the orthographic regularities of the writing system (Cohen et al., 2002; McCandliss et al., 2003). For example, the regional differences from the caudal to the more rostral part of the left PITC corresponds to the predisposed perceptual gradient from global to local processing (Ito et al., 1995; Lerner et al., 2001). Moreover, a recent fMRI study reported that, in Japanese adults, this region was selectively activated under a condition requiring new linkages between unfamiliar letters and speech sounds, indicating that this region shows dynamic functional plasticity even in adults (Hashimoto and Sakai, 2004). Thus, the left PITC might be involved in the visual word recognition that is a necessary subprocess of writing kanji by tuning into its orthographic regularities.

\section{Functional role of the right PITC}

In the mental recall of kanji orthography and during kana-tokanji transcription tasks, we found significantly shorter RTs after rTMS over the right (nondominant) PITC, whereas sham stimulation had no effect on RTs. Previous fMRI and magnetoencephalography studies have demonstrated weak activation or responses in the right PITC in some linguistic tasks, although this activation is much stronger on the left side (Nakamura et al., 2000; Cohen et al., 2002; Rossion et al., 2003). This strongly lateralized PITC activation may partially derive from the different functional importance of each hemisphere for language functions, including visual word recognition. A question then arises as to the nature of the functional role the right PITC might play in the processing of kanji.

In general, regarding the interhemispheric relationship in the language processing system, the dominant and nondominant hemispheres may work cooperatively or reciprocally suppress each other. There are two possible mechanisms by which the right PITC may contribute to kanji writing. One is that the right PITC plays a supplementary role to that of the left PITC, a hypothesis supported by tests on recovering aphasics and TMS studies (Basso et al., 1989; Knecht et al., 2002). The other is that the right PITC suppresses the function of the left PITC reciprocally based on the idea that the two hemispheres are equipotential for language functions at birth and that the language centers of one hemisphere become dominant by inhibiting homotopic areas in the other hemisphere via the corpus callosum during language acquisition (Netz et al., 1995; Karbe et al., 1998; Heiss et al., 2003). Based on the neural network model, Kinsbourne (2000) proposed that the two cerebral hemispheres are functionally equilibrate and reciprocally inhibit the areas that anchor them via corpus callosum (Kinsbourne, 2000). However, because lesion studies describing the effects of damage to the right hemisphere, including the PITC, are rare, the functional role of the right hemisphere remains unclear.

Recently, it has been reported that low-frequency rTMS is able to improve hemispatial neglect and nonfluent aphasia by the transient functional disruption of the corresponding area in the unaffected hemisphere, a phenomenon provisionally called paradoxical functional facilitation (Oliveri et al., 1999; Brighina et al., 2003; Martin et al., 2004). Moreover, other authors found similar paradoxical improvements in performances of hand movements or memory encoding after low-frequency rTMS over motor cortex or ventrolateral prefrontal cortex (Kobayashi et al., 2004; Kahn et al., 2005). A similar mechanism has been observed in animal models, in which a new lesion in the superior colliculus was shown to improve the visual dysfunction initially caused by an ipsilateral occipito-parietal lesion (Kapur, 1996; Lomber et al., 2002). The improvement of functional deficits by rTMS can be attributed to the normalization of the interhemispheric balance via the corpus callosum, through inhibiting the hyperactivation 
of the unaffected hemisphere by releasing the reciprocal inhibition from the affected hemisphere (Theoret et al., 2003). Previous anatomical study showed the existence of the interhemispheric anatomical connections between the inferior temporal gyri (including the PITC) through the corpus callosum in monkeys (Demeter et al., 1990).

TMS can elicit facilitation effect on the targeted cortical region and enhance its normal function on some occasions. When studying the carryover effect of rTMS, frequency-dependent suppression or facilitation has been observed in various languages related to cortical regions as well as motor cortex. Low-frequency rTMS at $1 \mathrm{~Hz}$ over just anterior to Broca's area prolonged RTs for verb production (Shapiro et al., 2001), whereas rTMS at $20 \mathrm{~Hz}$ over Wernicke's area reduced RTs in a picture-naming task (Mottaghy et al., 1999). Moreover, the event-related TMS caused reduction of RTs in object naming or syntactic decision tasks when TMS was administered to Wernicke's or Broca's area at a specific timing (Topper et al., 1998; Sakai et al., 2002). To our knowledge, there are no evidences for inducing facilitation effect on the targeted region by low-frequency rTMS $(<1 \mathrm{~Hz})$, except for a certain type of preconditioning enhancing the plasticity. For example, after the cathodal direct current stimulation for $10 \mathrm{~min}$ to the primary motor cortex, rTMS at $1 \mathrm{~Hz}$ can increase the corticospinal excitability (Siebner et al., 2004). However, these techniques are different from that used in the present study. Therefore, it is not likely that low-frequency rTMS over the nondominant PITC may directly facilitate its function.

Our results showing reduced RTs by transient disruption of the right PITC only in the tasks that require the visualization of kanji indicates that the functional suppressive action of the nondominant PITC might be relevant to the improved retrieval of kanji orthography. The right PITC might be functioning to tune into the orthographic regularities of kanji by inhibiting the left PITC when retrieving a complex mental image of the kanji configuration. It should be noted, however, that the interhemispheric language processing system might vary between the different regions and/or language functions.

\section{References}

Basso A, Gardelli M, Grassi MP, Mariotti M (1989) The role of the right hemisphere in recovery from aphasia: two case studies. Cortex 25:555-556.

Brighina F, Bisiach E, Oliveri M, Piazza A, La Bua V, Daniele O, Fierro B (2003) $1 \mathrm{~Hz}$ repetitive transcranial magnetic stimulation of the unaffected hemisphere ameliorates contralesional visuospatial neglect in humans. Neurosci Lett 336:131-133.

Chen R, Classen J, Gerloff C, Celnik P, Wassermann EM, Hallett M, Cohen LG (1997) Depression of motor cortex excitability by low-frequency transcranial magnetic stimulation. Neurology 48:1398-1403.

Clark VP, Keil K, Maisog JM, Courtney S, Ungerleider LG, Haxby JV (1996) Functional magnetic resonance imaging of human visual cortex during face matching: a comparison with positron emission tomography. NeuroImage 4:1-15.

Cohen L, Lehericy S, Chochon F, Lemer C, Rivaud S, Dehaene S (2002) Language-specific tuning of visual cortex? Functional properties of the visual word form area. Brain 125:1054-1069.

Dejerine J (1891) Sur un cas de cecite verbale avec agraphie, suivie d' autopsie. Comptes Rendus des Seances et Memoire de la Societe de Biologie 3:197-201.

Demeter S, Rosene DL, Van Hoesen GW (1990) Fields of origin and pathways of the interhemispheric commissures in the temporal lobe of macaques. J Comp Neurol 302:29-53.

Geschwind N (1965) Disconnexion syndromes in animals and man. I. Brain 88:237-294

Hashimoto R, Sakai K (2004) Learning letters in adulthood: direct visualization of cortical plasticity for forming a new link between orthography and phonology. Neuron 42:311-322.
Heiss WD, Thiel A, Kessler J, Herholz K (2003) Disturbance and recovery of language function: correlates in PET activation studies. NeuroImage 20 [Suppl 1]:S42-S49.

Ishai A, Ungerleider LG, Martin A, Schouten JL, Haxby JV (1999) Distributed representation of objects in the human ventral visual pathway. Proc Natl Acad Sci USA 96:9379-9384.

Ito M, Tamura H, Fujita I, Tanaka K (1995) Size and position invariance of neuronal responses in monkey inferotemporal cortex. J Neurophysiol 73:218-226.

Iwata M (1984) Kanji versus Kana: neuropsychological correlates of the Japanese writing system. Trends Neurosci 7:290-293.

Jahanshahi M, Rothwell J (2000) Transcranial magnetic stimulation studies of cognition: an emerging field. Exp Brain Res 131:1-9.

Kahn I, Pascual-Leone A, Theoret H, Fregni F, Clark D, Wagner AD (2005) Transient disruption of ventrolateral prefrontal cortex during verbal encoding affects subsequent memory performance. J Neurophysiol 94:688-698.

Kapur N (1996) Paradoxical functional facilitation in brain-behavior research-a critical review. Brain 119:1775-1790.

Karbe H, Thiel A, Weber-Luxenburger G, Herholz K, Kessler J, Heiss W (1998) Brain plasticity in poststroke aphasia: what is the contribution of the right hemisphere? Brain Lang 64:215-230.

Kawahata N, Nagata K, Shishido F (1988) Alexia with agraphia due to the left posterior inferior temporal lobe lesion-neuropsychological analysis and its pathogenetic mechanisms. Brain Lang 33:296-310.

Kawamura M, Hirayama K, Hasegawa K, Takahashi N, Yamaura A (1987) Alexia with agraphia of kanji (Japanese morphograms). J Neurol Neurosurg Psychiatry 50:1125-1129.

Kinsbourne M (2000) New models for old: taking the neural network seriously. Brain Cogn 42:13-16.

Knecht S, Floel A, Drager B, Breitenstein C, Sommer J, Henningsen H, Ringelstein EB, Pascual-Leone A (2002) Degree of language lateralization determines susceptibility to unilateral brain lesions. Nat Neurosci 5:695-699.

Kobayashi M, Hutchinson S, Theoret H, Schlaug G, Pascual-Leone A (2004) Repetitive TMS of the motor cortex improves ipsilateral sequential simple finger movements. Neurology 62:91-98.

Kosslyn SM, Pascual-Leone A, Felician O, Camposano S, Keenan JP, Thompson WL, Ganis G, Sukel KE, Alpert NM (1999) The role of area 17 in visual imagery: convergent evidence from PET and rTMS. Science 284:167-170

Lee L, Siebner HR, Rowe JB, Rizzo V, Rothwell JC, Frackowiak RS, Friston KJ (2003) Acute remapping within the motor system induced by lowfrequency repetitive transcranial magnetic stimulation. J Neurosci 23:5308-5318.

Lerner Y, Hendler T, Ben-Bashat D, Harel M, Malach R (2001) A hierarchical axis of object processing stages in the human visual cortex. Cereb Cortex 11:287-297.

Lomber SG, Payne BR, Hilgetag CC, Rushmore J (2002) Restoration of visual orienting into a cortically blind hemifield by reversible deactivation of posterior parietal cortex or the superior colliculus. Exp Brain Res 142:463-474.

Martin P, Naeser M, Theoret H, Tormos J, Nicholas M, Kurland J, Fregni F, Seekins H, Doron K, Pascual-Leone A (2004) Transcranial magnetic stimulation as complementary treatment for aphasia. Semin Speech Lang 25:181-191.

McCandliss BD, Cohen L, Dehaene S (2003) The visual word form area: expertise for reading in the fusiform gyrus. Trends Cogn Sci 7:293-299.

Moscovitch C, Kapur S, Kohler S, Houle S (1995) Distinct neural correlates of visual long-term memory for spatial location and object identity: a positron emission tomography study in humans. Proc Natl Acad Sci USA 92:3721-3725.

Mottaghy FM, Hungs M, Brugmann M, Sparing R, Boroojerdi B, Foltys H, Huber W, Topper R (1999) Facilitation of picture naming after repetitive transcranial magnetic stimulation. Neurology 53:1806-1812.

Mottaghy FM, Gangitano M, Sparing R, Krause BJ, Pascual-Leone A (2002) Segregation of areas related to visual working memory in the prefrontal cortex revealed by rTMS. Cereb Cortex 12:369-375.

Muellbacher W, Ziemann U, Boroojerdi B, Hallett M (2000) Effects of lowfrequency transcranial magnetic stimulation on motor excitability and basic motor behavior. Clin Neurophysiol 111:1002-1007. 
Nakamura K, Kouider S (2003) Functional neuroanatomy of Japanese writing systems. Aphasiology 17:667-683.

Nakamura K, Honda M, Okada T, Hanakawa T, Toma K, Fukuyama H, Konishi J, Shibasaki H (2000) Participation of the left posterior inferior temporal cortex in writing and mental recall of kanji orthography: a functional MRI study. Brain 123:954-967.

Netz J, Ziemann U, Homberg V (1995) Hemispheric asymmetry of transcallosal inhibition in man. Exp Brain Res 104:527-533.

Ogawa T, Inamura Y (1974) An analysis of word attributes imagery, concreteness, meaningfulness and ease of learning for Japanese nouns. Jpn J Psychology 44:317-327.

Oliveri M, Rossini PM, Traversa R, Cicinelli P, Filippi MM, Pasqualetti P, Tomaiuolo F, Caltagirone C (1999) Left frontal transcranial magnetic stimulation reduces contralesional extinction in patients with unilateral right brain damage. Brain 122:1731-1739.

Pascual-Leone A, Valls-Sole J, Wassermann EM, Hallett M (1994) Responses to rapid-rate transcranial magnetic stimulation of the human motor cortex. Brain 117:847-858.

Pascual-Leone A, Bartres-Faz D, Keenan J (1999) Transcranial magnetic stimulation: studying the brain-behaviour relationship by induction of "virtual lesions." Philos Trans R Soc Lond B Biol Sci 354:1229-1238.

Price CJ, Moore CJ, Humphreys GW, Frackowiak RS, Friston KJ (1996) The neural regions sustaining object recognition and naming. Proc R Soc Lond B Biol Sci 263:1501-1507.

Roeltgen D (1993) Agraphia. In: Clinical neuropsychology, Ed 3 (Heilman KM, Valenstein E, eds), pp 63-89. New York: Oxford UP.

Roeltgen D, Heilman K (1984) Lexical agraphia. Brain 107:811-827.

Roeltgen D, Sevush S, Heilman K (1983) Phonological agraphia: writing by the lexical-semantic route. Neurology 33:755-765.

Rosier A, Cornette L, Dupont P, Bormans G, Michiels J, Mortelmans L, Orban GA (1997) Positron-emission tomography imaging of long-term shape recognition challenges. Proc Natl Acad Sci USA 94:7627-7632.

Rossini PM, Barker AT, Berardelli A, Caramia MD, Caruso G, Cracco RQ, Dimitrijevic MR, Hallett M, Katayama Y, Lucking CH, Maertens de Noordhout AL, Marsden CD, Murray NM, Rothwell JC, Swash M, Tomberg C (1994) Non-invasive electrical and magnetic stimulation of the brain, spinal cord and roots: basic principles and procedures for routine clinical application. Report of an IFCN committee. Electroencephalogr Clin Neurophysiol 91:79-92.

Rossion B, Joyce CA, Cottrell GW, Tarr MJ (2003) Early lateralization and orientation tuning for face, word, and object processing in the visual cortex. NeuroImage 20:1609-1624.

Rounis E, Lee L, Siebner HR, Rowe JB, Friston KJ, Rothwell JC, Frackowiak RS (2005) Frequency specific changes in regional cerebral blood flow and motor system connectivity following rTMS to the primary motor cortex. NeuroImage 26:164-176.

Sakai KNY, Takeuchi T, Watanabe E (2002) Selective priming of syntactic processing by event-related transcranial magnetic stimulation of Broca's area. Neuron 35:1177-1182.

Sakurai Y, Sakai K, Sakuta M, Iwata M (1994) Naming difficulties in alexia with agraphia for kanji after a left posterior inferior temporal lesion. J Neurol Neurosurg Psychiatry 57:609-613.
Sakurai Y, Momose T, Iwata M, Sudo Y, Ohtomo K, Kanazawa I (2000) Different cortical activity in reading of Kanji words, Kana words and Kana nonwords. Brain Res Cogn Brain Res 9:111-115.

Satow T, Mima T, Yamamoto J, Oga T, Begum T, Aso T, Hashimoto N, Rothwell JC, Shibasaki H (2003) Short-lasting impairment of tactile perception by $0.9 \mathrm{~Hz}-\mathrm{rTMS}$ of the sensorimotor cortex. Neurology 60:1045-1047.

Sergent J, Ohta S, MacDonald B (1992) Functional neuroanatomy of face and object processing. A positron emission tomography study. Brain 115:15-36.

Shapiro KA, Pascual-Leone A, Mottaghy FM, Gangitano M, Caramazza A (2001) Grammatical distinctions in the left frontal cortex. J Cogn Neurosci 13:713-720.

Siebner HR, Lang N, Rizzo V, Nitsche MA, Paulus W, Lemon RN, Rothwell JC (2004) Preconditioning of low-frequency repetitive transcranial magnetic stimulation with transcranial direct current stimulation: evidence for homeostatic plasticity in the human motor cortex. J Neurosci 24:3379-3385.

Soma Y, Sugishita M, Kitamura K, Maruyama S, Imanaga H (1989) Lexical agraphia in the Japanese language. Brain 112:1549-1561.

Stewart L, Meyer B, Frith U, Rothwell J (2001) Left posterior BA37 is involved in object recognition: a TMS study. Neuropsychologia 39:1-6.

Theoret H, Kobayashi M, Valero-Cabre A, Pascual-Leone A (2003) Exploring paradoxical functional facilitation with TMS. Suppl Clin Neurophysiol 56:211-219.

Thuy DH, Matsuo K, Nakamura K, Toma K, Oga T, Nakai T, Shibasaki H, Fukuyama H (2004) Implicit and explicit processing of kanji and kana words and non-words studied with fMRI. NeuroImage 23:878-889.

Tokunaga H, Nishikawa T, Ikejiri Y, Nakagawa Y, Yasuno F, Hashikawa K, Nishimura T, Sugita Y, Takeda M (1999) Different neural substrates for Kanji and Kana writing: a PET study. NeuroReport 10:3315-3319.

Topper R, Mottaghy FM, Brugmann M, Noth J, Huber W (1998) Facilitation of picture naming by focal transcranial magnetic stimulation of Wernicke's area. Exp Brain Res 121:371-378.

Usui K, Ikeda A, Takayama M, Matsuhashi M, Yamamoto J, Satow T, Begum T, Mikuni N, Takahashi J, Miyamoto S, Hashimoto N, Shibasaki H (2003) Conversion of semantic information into phonological representation: a function in left posterior basal temporal area. Brain 126:632-641.

Valero-Cabre A, Payne BR, Rushmore J, Lomber SG, Pascual-Leone A (2005) Impact of repetitive transcranial magnetic stimulation of the parietal cortex on metabolic brain activity: a 14C-2DG tracing study in the cat. Exp Brain Res 163:1-12.

Walsh V, Cowey A (1998) Magnetic stimulation studies of visual cognition. Trends Cogn Sci 2:103-108.

Walsh V, Cowey A (2000) Transcranial magnetic stimulation and cognitive neuroscience. Nat Rev Neurosci 1:73-79.

Walsh V, Rushworth M (1999) A primer of magnetic stimulation as a tool for neuropsychology. Neuropsychologia 37:125-135.

Yamadori A (1975) Ideogram reading in alexia. Brain 98:231-238.

Yamadori A (2000) Neuropsychological model of reading based on Japanese experiences. Psychologia 43:1-14. 